\title{
Anesthesia management in Joubert Syndrome: a case report
}

\author{
Baki ED*, Bezen BA and Yüksek A \\ Afyon Kocatepe University, Departmant of Anesthesiology and Reanimation, Turkey
}

\begin{abstract}
Joubert syndrome is a rare autosomal recessive disorder of the cerebellum that occurs in 1 of 100,000 live births. The syndrome is characterized by malformations of the cerebellum and brain stem, hypotonia, developmental delay, hyperpnea or apnea attacks or atypical eye movements. Cognitive changes are mild to severe, and can range to the extent of mental retardation. These patients may be sensitive to respiratory depression caused by anesthetics, so the anesthetic management of these patients needs more attention. The case is here presented of the anesthesia management of a 5-year-old girl with Joubert Syndrome who underwent general anesthesia for surgery to a supracondylar humerus fracture.
\end{abstract}

\section{Introduction}

Joubert syndrome (JS) is an inherited autosomal recessive disorder in which the cerebellar vermis is hypo or aplasic [1]. In the neonatal period, it is characterized by hypotony, tachypnea and apnea episodes, abnormal eye and tongue movements, ataxia, and mental-motor retardation accompanies the extracerebral signs in some patients [1,2]. Axial cranial MR images of patients with Joubert syndrome show a molar tooth appearance and a bat wing appearance on axial and coronal cranial sections [3]. These patients may be susceptible to respiratory depression with anesthetic agents (especially opioids and neuromuscular blockade agents) [2-5]

The anesthesia management is presented here of a 5-year-old girl with Joubert Syndrome who underwent general anesthesia for surgery to a supracondylar humerus fracture.

\section{Case report}

A 5-year old girl weighing $25 \mathrm{~kg}$ was admitted to the Orthopedics Department with a supracondylar humerus fracture following a fall. The patient had been delivered by caesarean section at 41 weeks gestation from the first pregnancy of her mother. The pregnancy had been normal and there was no consanguinity between the parents. The patient was hypotonic at birth and stayed in the Intensive Care Unit for 13 days because of respiratory disorders at birth. Heart, kidney and brain pathologies were detected prenatally and Joubert Syndrome was diagnosed with cranial magnetic resonance imaging (MRI) at the age of 6 months. The physical examination revealed hypertelorism, a large tongue, and high palate. There was no anesthesia history other than sedation during MRI. The Mallampati score was class 1 and laboratory test results were normal. Informed consent was obtained from the parents and the patient was taken to the operating room. Intravenous access was maintained easily with perfect cooperation and premedication was administered of midazolam $(0.007 \mathrm{mg} / \mathrm{kg})$. Following routine anesthesia monitoring, ketamine $25 \mathrm{mg}(1 \mathrm{mg}$ $/ \mathrm{kg})$ propofol $50 \mathrm{mg}(2 \mathrm{mg} / \mathrm{kg})$ and rocuronium $15 \mathrm{mg}(0.6 \mathrm{mg} /$ $\mathrm{kg}$ ) were administered and she was intubated with a 5- uncuffed endotracheal tube without any problems. Anesthesia was maintained with 1 MAC (minimum alveolar concentration) sevoflurane in 50\% oxygen- $50 \%$ air mixture. The surgical procedure lasted $30 \mathrm{~min}$. After spontaneous breathing of the patient, sugammadex $50 \mathrm{mg}(2 \mathrm{mg} / \mathrm{kg})$ was administered and extubation was applied smoothly. Postoperative analgesia of $250 \mathrm{mg}$ paracetamol $(10 \mathrm{mg} / \mathrm{kg})$ was administered and the patient was transferred to the postanesthesia care unit (PACU). Under close monitoring, there was no apneic or hyperpneic periods during follow up in PACU. When the patient was considered stable, she was transferred to the Orthopedic ward and discharged from the hospital 36 hours postoperatively.

\section{Discussion}

Joubert syndrome (JS) was first described by Marie Joubert, a pediatric neurologist in Montreal in 1969 [2-6]. It is now known that JS is due to structural and/or functional impairment of primary silica [7].

It is seen in the neonatal period, as a complicated disease with respiratory abnormalities characterized by tachypnea-apnea episodes and cerebellar vermis disorders, and occasionally accompanied by cardiac, renal and ocular disorders [2-5]. In the current case, in addition to cardiac and renal pathologies in the prenatal period, there was a history of neonatal intensive care unit hospitalization due to hypotonia and respiratory defects. The humerus fracture was the result of a fall caused by loss of balance while walking, which can be a sign that the cerebellar vermis is affected.

There have been few reports about the anesthetic management of Joubert syndrome. The first report was about inguinal hernia repair in an infant [8]. General anesthesia was maintained with thiopentone,

Correspondence to: Baki ED, Associate Prof, Afyon Kocatepe University, Faculty of Medicine, Anesthesiology and Reanimation Department, Campus of Ali Çetinkaya, Afyon-İzmir Karayolu 8.km, Afyonkarahisar, 03200, Turkey. Tel: +90 5308739908; Email: elifbaki1973@mynet.com,

Key words: Sugammadex, Joubert Syndrome, apnea, anesthesia

Received: November 29, 2017; Accepted: December 23, 2017; Published: December 26, 2017 
$\mathrm{N} 2 \mathrm{O}, \mathrm{O} 2$, isoflurane, and many apneic episodes were experienced by the patient which persisted for several hours [8]. Patients with Joubert syndrome may be susceptible to respiratory depression with anesthetic agents (especially opioids and neuromuscular blocking drugs) [2-9]. It has been emphasized in literature that muscle relaxants and opioids should be avoided in the anesthesia management of this syndrome. Güclü et al reported the case of a 7-year old child with Joubert syndrome who underwent surgery for inguinal hernia, hydrocele and circumcision [10]. Sevoflurane and propofol were used for anesthesia maintenance, no neuromuscular blocking agents and opiods were used, and penile block was applied for analgesia [10]. Vodopich et al used spinal anesthesia under propofol sedation with spontaneous ventilation for inguinal hernia repair on a child with Joubert syndrome [9]. Although not recommended in literature, muscle relaxant was used in the anesthesia induction of the current case, anesthesia was maintained with sevoflurane, and ketamine was used instead of opioids for intraoperative analgesia. The ease of use of muscle relaxants was due to having a strong antidote. Sugammadex is an agent used in the reversal of muscle relaxation with some non-depolarizing neuromuscular blocking drugs [4]. Its effect is shown by binding and removing vecuronium and rocuronium from the plasma and as it binds to rocuronium at a ratio of $1: 1$, it is rapidly eliminated [4]. In the current case, sugammadex was used at a dose of $2 \mathrm{mg} / \mathrm{kg}$, and no apneic episodes occurred during the recovery period of the patient.

The presence of anatomic findings in the JS such as cleft palate, large tongue, small jaw, and laryngomalacia can complicate airway management and can make intubation difficult [10]. The Mallampati score of the currrent patient was class 1 and as mask ventilation was easy, there was no difficulty in intubation.

Joubert Syndrome is one of the diseases for which management of anesthesia can be difficult. A careful pre-anesthetic evaluation for safe anesthesia and a competent understanding of the possible adverse effects of the disease will reduce peri-operative problems [6]. The use of sugammadex in Joubert Syndrome can be considered to improve the recovery period after general anesthesia.

\section{References}

1. Paksu MŞ, Dağdemir A, Taşdemir HA, Güngör O, Küçüködük Ş, et al. (2004) Joubert syndrome case report Journal of Ondokuz Mayls University Medical Faculty 21: 9093. [Crossref]

2. Bhaskar P, John J, Sivamurthy SK et al (2013) Anesthetic management of an infant with Joubert syndrome for cardiac surgery. $J$ Clin Anesth 25: 488-90. [Crossref]

3. Sarac V, Yazıcı Z, Aktürk C, Erdoğan C, Okan M et al. (2004) c Journal of Uluda $\breve{g}$ University Medical Faculty 30: 119.

4. Canpolat GD, Yıldırım MD, Doğan S, Doğruel F, Per H (2017) Sugammadex and General Anesthesia in Two Pediatric Patients with Joubert Syndrome: Case Report. Erciyes Med J 39: 35-6.

5. Habre W, Sims C, D'Souza M (1997) Anaesthetic management of children with Joubert syndrome. Paediatr Anaesth 7:251-3. [Crossref]

6. Sriganesh K, Vinay B, Jena S, Sudhir V, Saini J (2014) Anesthetic management of patients with Joubert syndrome: a retrospective analysis of a single-institutional case series. Paediatr Anaesth 24: 1180-1184. [Crossref]

7. Buntenbroich S, Dullenkopf A (2013) Total intravenous anesthesia in a patient with Joubert-Boltshauser syndrome. Paediatr Anaesth 23: 204-205. [Crossref]

8. Matthews NC (1989) Anaesthesia in an infant with Joubert's syndrome. Anaesthesia 44: $920-921$.

9. Vodopich DJ, Gordon DJ (2004) Anesthesia management in Joubert syndrome Paediatr Anaesth 14: 871-873.

10. Matthews NC (1989) Anaesthesia in an infant with Joubert's syndrome. Anaesthesia 44: $920-921$.

Copyright: (C2017 Baki ED. This is an open-access article distributed under the terms of the Creative Commons Attribution License, which permits unrestricted use, distribution, and reproduction in any medium, provided the original author and source are credited. 